\title{
Conditioned Pain Modulation (CPM) Effects Captured in Facial Expressions
}

This article was published in the following Dove Press journal:

Journal of Pain Research

\author{
Miriam Kunz' \\ Stefanie F Bunk (D) ${ }^{2}$ \\ Anna J Karmann ${ }^{3}$ \\ Karl-Jürgen Bär ${ }^{4}$ \\ Stefan Lautenbacher ${ }^{3}$ \\ 'Department of Medical Psychology and \\ Sociology, University of Augsburg, \\ Augsburg, Germany; ${ }^{2}$ Department of \\ General Practice and Elderly Care \\ Medicine, University Medical Center \\ Groningen, University of Groningen, \\ Groningen, The Netherlands; \\ ${ }^{3}$ Physiological Psychology, Otto-Friedrich \\ University Bamberg, Bamberg, Germany; \\ ${ }^{4}$ Department of Psychosomatic Medicine \\ and Psychotherapy, University Hospital, \\ Jena, Germany
}

Purpose: Conditioned pain modulation (CPM) is most often assessed using self-report of pain. However, self-report of pain is not always available (eg in individuals with cognitive impairment) and is susceptible to report bias. In comparison, the facial expression of pain is more reflex-like and represents one of the most sensitive and specific non-verbal signals of pain. The aim of the present study was to investigate whether the facial expression of pain is sensitive enough to capture endogenous pain inhibition as elicited during CPM paradigms.

Patients and Methods: In total, 26 female participants took part in this study. Facial and verbal responses to phasic heat pain were assessed once while participants immersed their hand in a hot water bath and once without additional stimulation. Facial responses were analyzed using the Facial Action Coding System (FACS). Verbal responses were assessed using a Numerical Rating Scale (NRS).

Results: Pain-relevant facial responses as well as pain ratings to phasic heat pain were significantly reduced when participants simultaneously immersed their hand in a hot water bath compared to baseline. Thus, CPM effects could be demonstrated both on subjective as well as on facial responses. Moreover, CPM-induced changes in pain-relevant facial responses and in NRS ratings were significantly correlated.

Conclusion: The present study shows that facial expressions of pain are sensitive enough to capture CPM effects. Given the proven clinical usefulness of assessing CPM, the parallel assessment of verbal and facial CPM effects might be a promising approach with wider scope of applications. Further research in other demographic healthy participant and clinical cohorts is warranted.

Keywords: pain, CPM, conditioned pain modulation, facial expression, FACS

\section{Introduction}

The study of pain inhibition by use of the so-called conditioned pain modulation (CPM) paradigm has become a major focus of interest in clinical pain research. The CPM paradigm refers to an experimental procedure that examines the extent to which pain responses to phasic stimuli (test stimuli) are reduced by the concurrent application of a tonic stimulus (conditioning stimulus) applied to a remote area of the body. There does not exist one gold-standard CPM protocol, but there is much variability with regard to the mode of application of the "test" as well as the "conditioning" stimuli. Despite the methodological variability, greater reductions in pain responses are interpreted as greater endogenous pain inhibition. There is clear evidence that CPM responses are reduced in many chronic pain syndromes ${ }^{1,2}$ such as chronic low back pain, ${ }^{3}$ irritable bowel syndrome ${ }^{4}$ chronic orofacial pain, ${ }^{5}$ fibromyalgia, ${ }^{6}$ migraine ${ }^{7,8}$ and chronic tension-type headache, ${ }^{9}$ although it is still
Department of Medical Psychology and Sociology, University of Augsburg,

Augsburg, Germany

Tel $+49821598-3720$

Fax $+49821598-5116$

Email Miriam.kunz@med.uni-augsburg.de 
not definitely established whether the chronic pain is consequence or cause of the changed CPM. Further studies addressed whether changes in CPM mediates or predicts anti-nociceptive effects of certain forms of pain management ${ }^{1,10-12}$ while others targeted the analgesic effects of certain drugs and alcohol. ${ }^{13-16}$ Based on these findings, there is growing interest to use CPM paradigms in clinical practice for pain diagnostics and mechanismbased treatment (eg to predict analgesic responses or the risk for developing postoperative pain). ${ }^{17,18}$ Given its potential clinical relevance, it is not surprising that nonpathological variations of CPM due to age ${ }^{19}$ sleep $^{8,20}$ personality, ${ }^{21}$ and $\operatorname{sex}^{22}$ have also been found to affect CPM.

Most of the studies have applied psychophysical protocols, where changes in self-report ratings of the test stimulus are used as indicators of potential CPM effects. Relying solely on self-report ratings, however, limits the CPM assessment to vigilant and cognitively healthy individuals. There are methodological alternatives, which do not require self-report ratings, with the nociceptive flexion reflex (NFR) being the most frequently used alternative method to assess CPM inhibition. ${ }^{23}$ Moreover, in a few recent studies, nociceptive brain potentials were also used as an alternative method for CPM inhibition. ${ }^{24-26}$ NFR and brain potentials can, however, only be elicited by very brief stimuli as produced by electrical current, short heat pulses and nasal gas puffs, that show little resemble to clinical pain states.

To widen the methodological scope of CPM protocols, the aim of the present study was to test - for the first time - whether behavioral pain responses may also show CPM inhibition. In contrast to NFR and brain potentials, behavioral responses have the advantage that they can be elicited using a wider range of pain stimuli of various length; and thus, by stimuli that might show greater resemblance to clinical pain states. Facial responses to pain are the most prominent behavioral indicator of pain and they allow for fine-grained graduations both of clinical and experimental pain. ${ }^{27}$ In case of experimental pain, various modalities (heat, cold, pressure) and durations (phasic, tonic) can be used to reliably evoke facial activity. ${ }^{27}$ The question under study was whether CPM effects can be inferred by using facial responses as outcome measures. We expected similar CPM effects for facial and for verbal responses, although facial and verbal responses to pain have rarely been shown to be strictly coupled. ${ }^{28}$ Thus, the inhibitory effects on the facial responses were expected to be overlapping but yet independent from verbal responses. We created ideal conditions for this first test of a "facial CPM paradigm" by only studying women, which have been shown to facially express pain in a more fine-grained manner than men. ${ }^{29}$

\section{Participants and Methods}

\section{Participants}

We recruited 27 female participants (age: mean=24.1; $\mathrm{SD}=4.4$ ) by bulletins put up throughout the campus of the University of Jena. Sample size calculation (GPower 3.1) was based on previous studies ${ }^{14,30,31}$ and on our interest in "medium" effects that might be of clinical relevance (sample size calculation were conducted for $80 \%$ power and 0.05 level of significance; resulting in the above mentioned $\mathrm{N}$ of 27 subjects). All participants underwent a screening to test for any psychiatric disorders using the German version of the Mini-International Neuropsychiatric Interview (MINI) ${ }^{32}$ and a semistructured interview for recording past and current neurological as well as psychiatric symptoms and disorders. Moreover, all participants were pain free and all participants were right-handed. The present experiment was part of a larger study on the effect of tryptophan depletion on cognitive performances, brain activation and pain. ${ }^{33}$ Data of the present experiment were assessed during the pharmacological control condition. The study complied with the Declaration of Helsinki. Informed written consent was obtained in accordance with the protocols approved by the Ethics Committee of the University of Jena and all participants received monetary compensation. All participants were blind to the study hypothesis. The individual in Figure 1 provided informed consent for her photos to be published.

\section{Design}

The testing took place at the Department of Psychosomatic Medicine and Psychotherapy of the, University Hospital Jena. The experimental procedure used to assess CPM effects was divided into three blocks (see Figure 2). In the first block, the individual heat pain threshold was assessed. In blocks 2 and 3, phasic heat pain (tailored to the pain threshold) was applied once without (baseline block) and once with concurrent tonic heat pain stimulation (CPM block). Facial and verbal responses to the phasic and tonic pain stimulation were assessed (see Figure 2). 

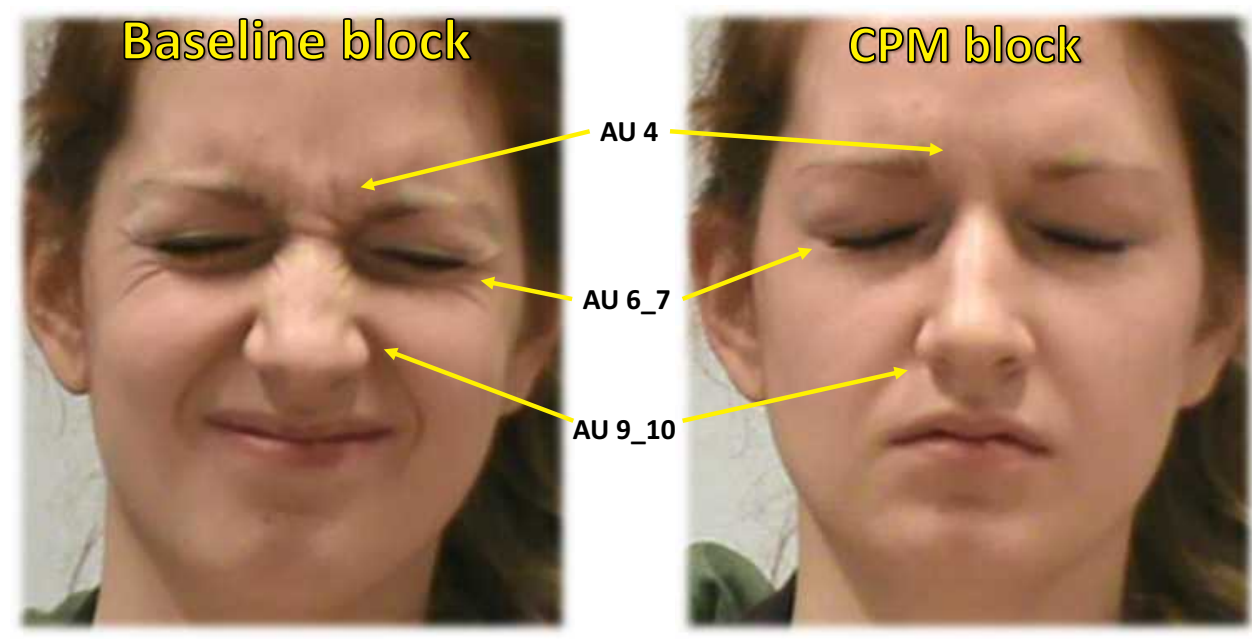

Figure I Examples of pain-relevant facial responses to the phasic heat pain (test stimuli) assessed during baseline block and the CPM block.

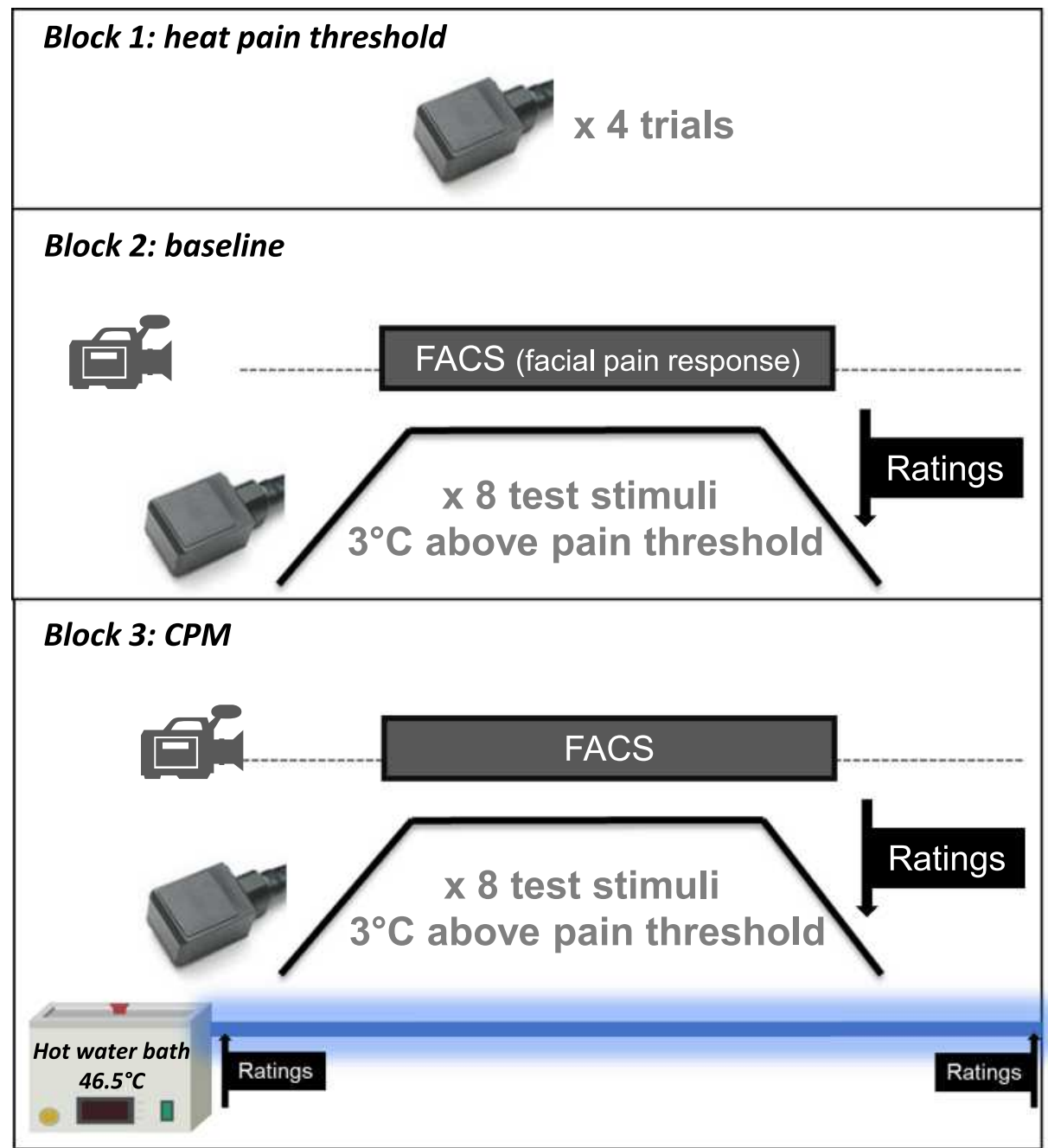

Figure 2 Graphical overview of the study protocol. 


\section{Block I: Heat Pain Thresholds}

Thermal stimulation was applied on the tibia of the left leg (centrally in between knee and ankle) by a Peltier based contact stimulation device (Medoc, TSA-2001, Ramat Yishai, Israel) with a $30 \mathrm{~mm} * 30 \mathrm{~mm}$ contact thermode that was attached to the leg via an elastic bandage. Heat pain thresholds were determined using the method of limits. The temperature increased from $35^{\circ} \mathrm{C}$ at a rate of $1{ }^{\circ} \mathrm{C} / \mathrm{s}$ and participants were asked to press a mouse button as soon as the temperature reached a level that was barely painful. Each time they pressed the button, the temperature returned to $35^{\circ} \mathrm{C}\left(5^{\circ} \mathrm{C} / \mathrm{s}\right)$ and the next trial started after an interstimulus interval of $9-11$ seconds. Following two familiarization trials, there were four trials and the average of these trials was used to constitute the threshold estimate.

\section{Block 2 and 3: Assessment of Conditioned Pain Modulation (CPM)}

CPM was assessed with thermal stimuli as conditioning (hot water immersion) as well as test (thermode) stimulus. This kind of parallel combination of hot water immersion for conditioning stimulation and thermode application for test stimulation has been well established in our laboratory and shown robust inhibitory effects in several studies for psychophysical data. ${ }^{4,30,31}$

\section{Test Stimuli}

The test stimuli were applied on the tibia of the left leg by the above described Peltier-based contact stimulation device. The intensities were tailored to the individual threshold $\left(+3^{\circ} \mathrm{C}\right.$ above threshold) to ensure that the stimulation would be perceived as painful but tolerable. During each block, eight test stimuli were applied. The temperature increased from baseline $\left(38^{\circ} \mathrm{C}\right)$ with a rate of $5 \% \mathrm{~s}$ until it reached the target temperature, and remained at a plateau for $5 \mathrm{~s}$, before returning to baseline. A long and variable interstimulus interval (ISI) between 12 and $15 \mathrm{~s}$ was used to prevent sensitization and to allow subjects to rate each stimulus. The eight test stimuli were first applied alone (block 2), and then in combination with the conditioning stimulus (block 3).

\section{Conditioning Stimulus}

As a conditioning stimulus, we used tonic heat stimulation, which was realized by hand immersion in a circulation water bath (Witeg $\mathrm{GmbH}$, WiseCircu WCB-22, Wertheim, Germany) containing water at a temperature of $46.5{ }^{\circ} \mathrm{C}$. The participant immersed the right hand up to $2 \mathrm{~cm}$ above the wrist in this water bath. Water temperature was controlled with a thermostat, and the water was stirred with a force and suction pump to avoid layers of lower temperature around the hand. The immersion time of the hand into the water bath was as long as necessary to apply all eight test stimuli, which took around 4.5 minutes (test stimuli started after 30 seconds of hand immersion).

\section{Pain Responses \\ Self-Report Ratings}

After the application of each test stimulus, participants were asked to verbally rate the perceived intensity using a numerical rating scale (NRS; 0-100). Participants were instructed that "0" corresponded to "no pain" and " 100 " to "extremely strong pain", respectively. With regard to the conditioning stimulus, participants were asked at the beginning (after 10 seconds immersion time) as well as the end of hand immersion to verbally rate the pain intensity caused by water immersion on the same NRS $(0-100)$.

\section{Facial Pain Responses}

The face of the participants was videotaped throughout blocks 2 and 3. The camera was located approximately $2.0 \mathrm{~m}$ from the participant. In order to mark the plateau phase of the stimuli, a LED visible to the camera, but not to the participant, was lit concurrently with the test stimuli, starting when the target temperature was reached. During stimulation, participants were instructed not to talk and to look at a cross on the wall behind the camera to ensure that the face would always be recorded in an upright and frontal view.

Facial expressions were coded from the video recordings using the Facial Action Coding System $\left(\right.$ FACS $^{34}$ ), which is based on anatomical analysis of facial movements and distinguishes 44 different Action Units (AUs) produced by single muscles or combinations of muscles. A certified FACS coder (qualified by passing an examination given by the developers of the system), who was blind to the condition, identified the frequency and the intensity (5-point scale) of the different Action Units. In addition, $10 \%$ of the video data was also coded by a second certified observer and interrater reliability (using the EkmanFriesen formula ${ }^{34}$ ) was 0.81 , which compares favorably 
with other research in the FACS literature. ${ }^{35,36} \mathrm{~A}$ software designed for the analysis of observational data (the Observer Video-Pro; Noldus Information Technology) was used to segment the videos and to enter the FACS codes into a time-related database. Time segments of $7 \mathrm{~s}$ ( 5 $\mathrm{s}$ of plateau $+2 \mathrm{~s}$ return to baseline) were selected for scoring. In total, 16 segments of phasic pain stimulation (8 test stimuli $\mathrm{x} 2$ blocks) were analyzed in each participant. For the purpose of necessary data reduction, we combined similar facial responses as has been done in preceding studies without any loss of information. ${ }^{36-39}$ Those combinations include AU1_2, AU6_7, AU9_10 and AU25_26_27. The percentage of occurrence of each AU was calculated across the 16 segments of phasic pain stimulation and are shown in Figure 3 (AUs that were never scored are not displayed).

For further analyses, we focused only on pain-relevant AUs, which are based on a recent review article on facial expressions of pain $^{27}$ and include AU4 (contraction of the eyebrows), AU6_7 (contraction of the muscles surrounding the eyes), AU9_10 (nose wrinkle/upper lip raise) and AU 26_26_27 (opening of the mouth). As can be seen in Figure 2, these also were the most frequent AUs in the present study. AU-frequency and AU-intensity values for each of these pain-relevant AUs were combined (product terms) and then square root transformed, due to the fact that the values were not distributed normally (Kolmogorov-Smirnov p-values $<0.05$ ).

\section{Analyses}

CPM effects on subjective and facial responses were analyzed using analyses of variance with repeated measurements (comparing responses to the test stimuli in the baseline block vs CPM block; ANOVA for verbal responses, MANOVA for facial responses). Moreover, we used correlation analysis to assess whether there is an association between subjective and facial CPM effects. Analyses were conducted using SPSS 26 and findings were considered to be statistically significant at $\alpha<0.05$.

\section{Results}

The average heat pain threshold was $45.01^{\circ} \mathrm{C}$ (SD 1.07). Given that the test stimuli were tailored to the individual pain threshold $\left(+3^{\circ} \mathrm{C}\right.$ above threshold (there was one participant where the temperature could only be increased $+2.9^{\circ} \mathrm{C}$ due to the upper temperature limit of $50^{\circ} \mathrm{C}$ )), the average temperature of the test stimuli correspondingly

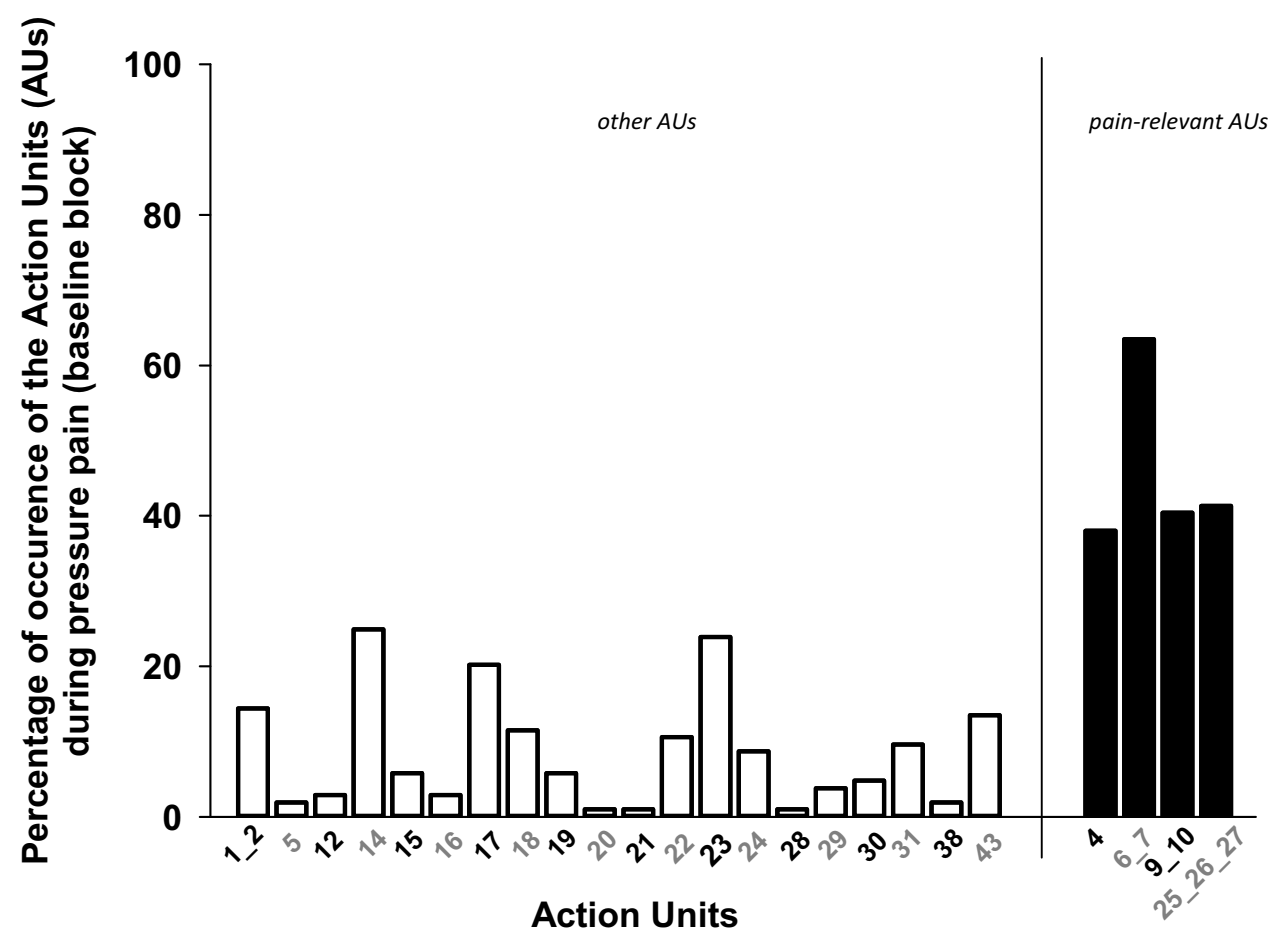

Figure 3 Percentage of occurrence of facial action units (AUs) across all painful stimuli (combining baseline and CPM blocks). 
was $48.01{ }^{\circ} \mathrm{C}\left(\mathrm{SD} 1.07^{\circ} \mathrm{C}\right.$ ) (intensities ranging from $45.6^{\circ}$ $\mathrm{C}$ to $50.0^{\circ} \mathrm{C}$ ).

The hot water immersion conditioning stimulus (stimulus intensity was set to $46.5^{\circ} \mathrm{C}$ ) was rated as moderately painful (mean NRS rating of $61.6( \pm 26.9)$ at the beginning and $65.4( \pm 27.8)$ at the end $(\mathrm{p}<0.01$ between ratings $))$. In order to ensure, that facial responses during the CPM paradigm (phasic heat) were not confounded by facial responses to the hot water immersion, we also inspected facial expressions occurring in response to the hot water immersion. Although facial expressions of pain were elicited by the hot water immersion, these facial responses were sparse and of low intensity and almost never $(<3 \%)$ occurred right before the onset of the test stimulus (thermode) (3 seconds before start).

\section{Conditioned Pain Modulation (CPM) Self-Report Rating}

As can be seen in Figure 4A, pain ratings of the phasic heat stimuli significantly decreased when participants simultaneously immersed their hand in a hot water bath compared to no concurrent pain stimulation $(\mathrm{F}(1,25)=6.31$, $\mathrm{p}=0.019$; effect size Cohen's d: 0.73). Thus, we found a significant CPM effect of moderate effect size on pain ratings. To determine whether this CPM effect might depend on the individual test stimulus temperature, we correlated the CPM effect (change in the NRS scores) with the test stimulus temperature and found no significant association $(\mathrm{r}=0.287, \mathrm{p}=0.155)$.

\section{Facial Response}

The MANOVA yielded a significant multivariate CPM effect $(\mathrm{F}(4,22)=3.15, \mathrm{p}=0.034$; effect size partial eta squared $\left.\eta^{2}: 0.364\right)$. As can be seen in Figure 4B, facial responses to phasic heat pain significantly decreased when participants simultaneously immersed their hand in a hot water bath. As regards the univariate outcomes, we found significant CPM effects for contraction of the eyebrows (AU4; $p=0.014$; Cohen's d: 0.76 ), contraction of the muscles surrounding the eyes (AU6_7; $\mathrm{p}=0.010$; Cohen's d: 0.79) and lifting of the upper lip (AU9_10; $p=0.018$; Cohen's d: 0.78), whereas no significant CPM effect was found for opening of the mouth (AU25_26_27; $\mathrm{p}=0.125$; Cohen's d: 0.44). Thus, we found significant CPM effects of moderate to large sizes inferred by 3 out of the 4 painrelevant facial responses. Figure 1 gives an individual example of the CPM effects inferred by facial responses.
To determine whether the significant CPM effects might depend on the individual test stimulus temperature, we correlated the CPM effect (change in the pain-relevant composite AU score) with the test stimulus temperature and found no significant association $(r=0.030, \mathrm{p}=0.870)$.

In an additional step, we wanted to investigate whether the CPM effects on self-report ratings and on facial responses, respectively, are independent enough, so that they prevail even when controlling for the other type of response.

\section{Self-Report (Covariate Facial Response)}

When entering the CPM effect on pain-relevant facial responses (change in the pain-relevant composite score) as a covariate into the analysis of variance, no significant CPM effects on self-report ratings could be found $(\mathrm{F}=1,24)=0.635, \mathrm{p}=0.433$; effect size $\eta^{2}: 0.026$ (small effect).

\section{Pain-Relevant Facial Response (Covariate Self-Report)}

When entering the CPM effect on self-report ratings (change in the NRS scores) as a covariate into the analysis of variance, the significant CPM effect on pain-relevant facial responses remained $(F(1,24)=5.859, p=0.023$; effect size $\eta^{2}: 0.202$ (large effect)).

\section{Correlation Between Self-Report and Facial CPM Effect}

In a last step, we wanted to investigate whether there is an association between CPM effects on self-report ratings and on facial responses. Correlation analysis showed a positive, moderate association between CPM effects on self-report ratings and on pain-relevant facial responses (composite score) $(\mathrm{r}=0.449, \mathrm{p}=0.021)$. As can be seen in Figure 5, greater CPM effects on self-report ratings were associated with greater CPM effects on pain-relevant facial responses.

\section{Discussion}

In the present study, CPM effects were for the first time documented by assessing the reactions to the test stimuli through recording facial responses and not - as typically done - by means of self-report. What was the reason for looking for such behavioral alternatives? The motivation of this endeavor was to develop a protocol that does not depend on the individual being vigilant, cognitively healthy and/or linguistically competent. The presented 


\section{A Self-report ratings}

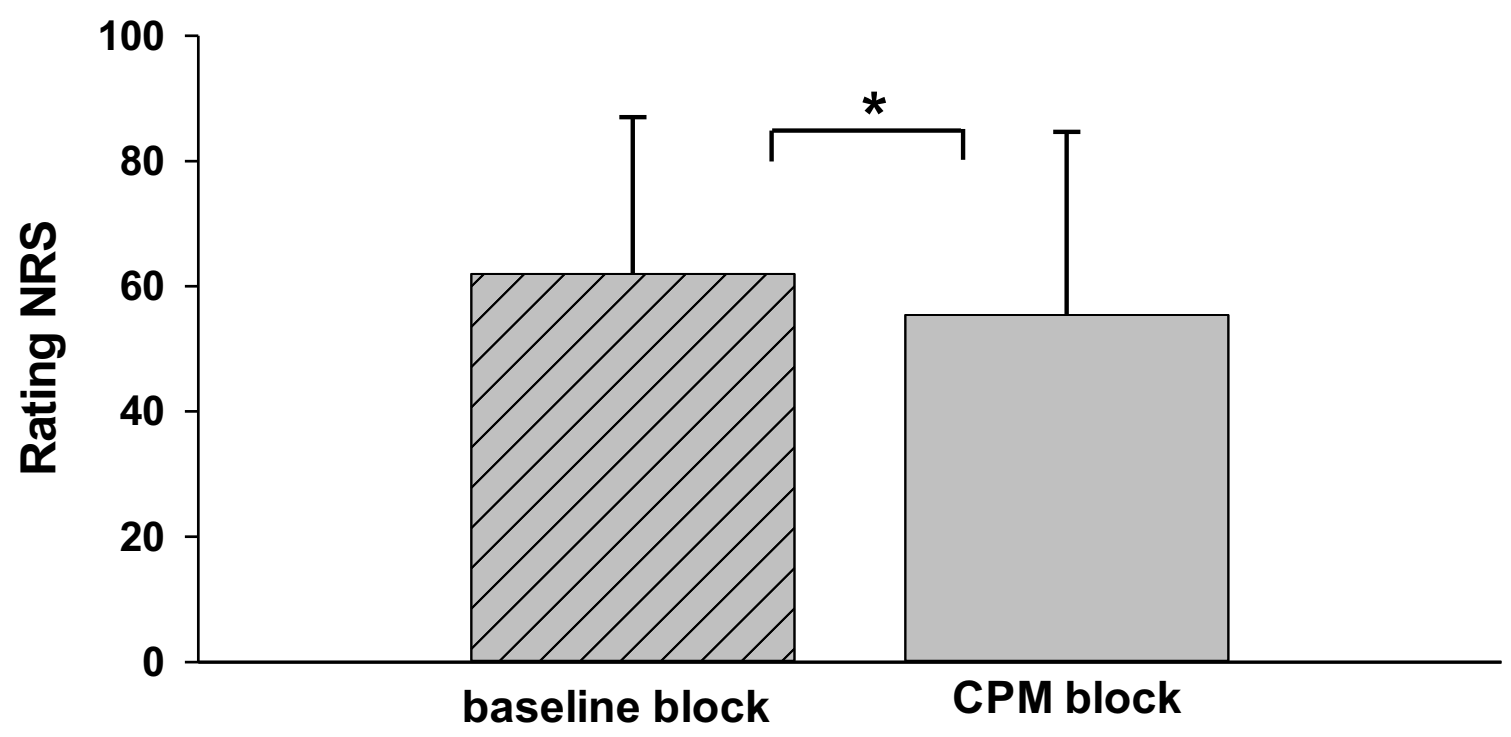

B Pain-relevant facial responses

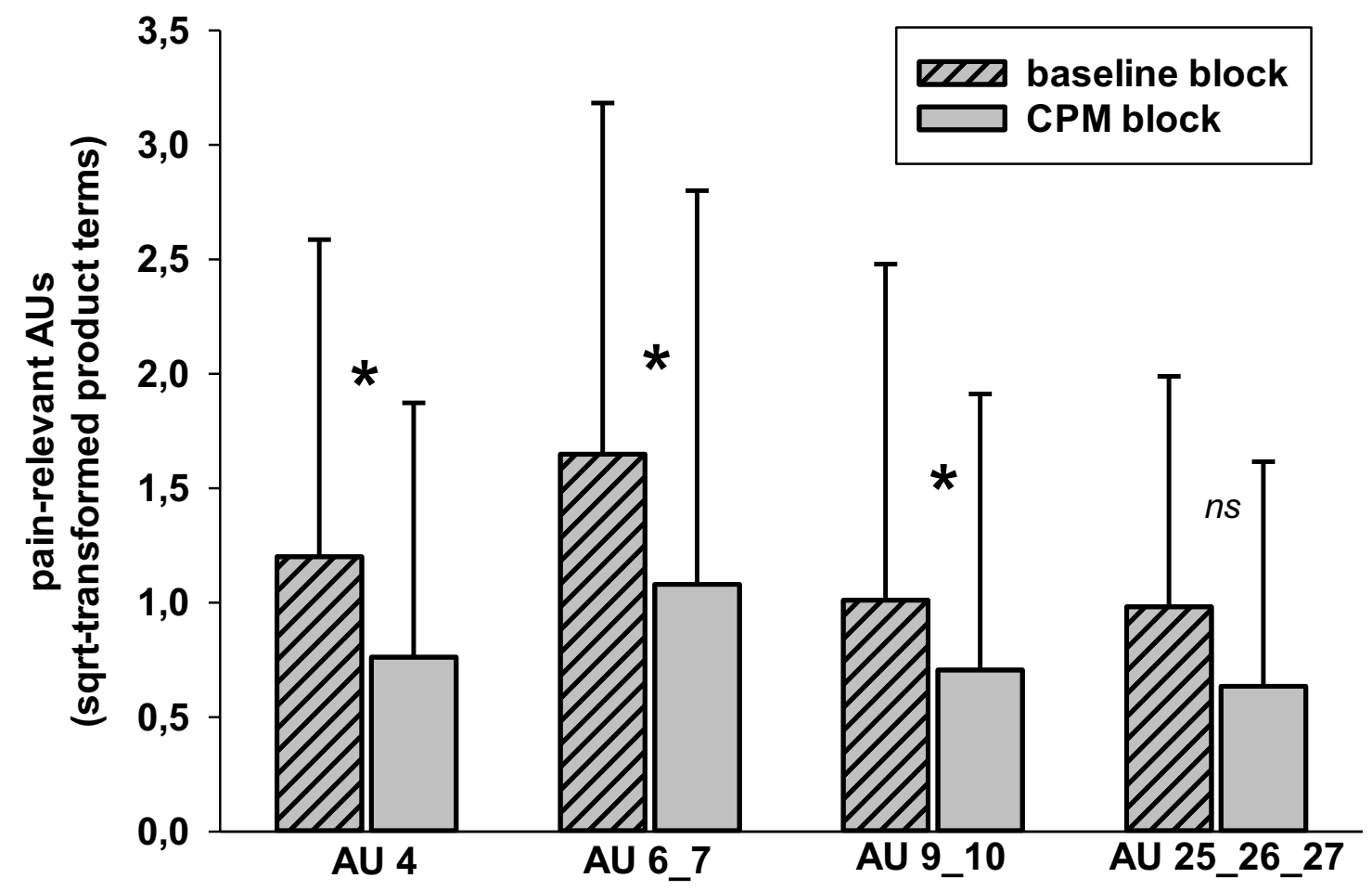

AU4: contraction of the eyebrows; AU6_7: contraction of the muscles surrounding the eyes; AU9_10: nose wrinkle/ upper lip raise; AU 26_26_27: opening of the mouth * indicates $\mathrm{p}<0.05, \mathrm{~ns}=$ not significant

Figure 4 CPM effect on self-report ratings (A) and on pain-relevant facial responses (B). Mean responses (and SD) are given for the baseline block and the CPM block. Note: *Indicates $\mathrm{p}<0.05$, ns= not significant.

Abbreviations: AU4, contraction of the eyebrows; AU6_7, contraction of the muscles surrounding the eyes; AU9_10, nose wrinkle/upper lip raise; AU 26_26_27, opening of the mouth. 


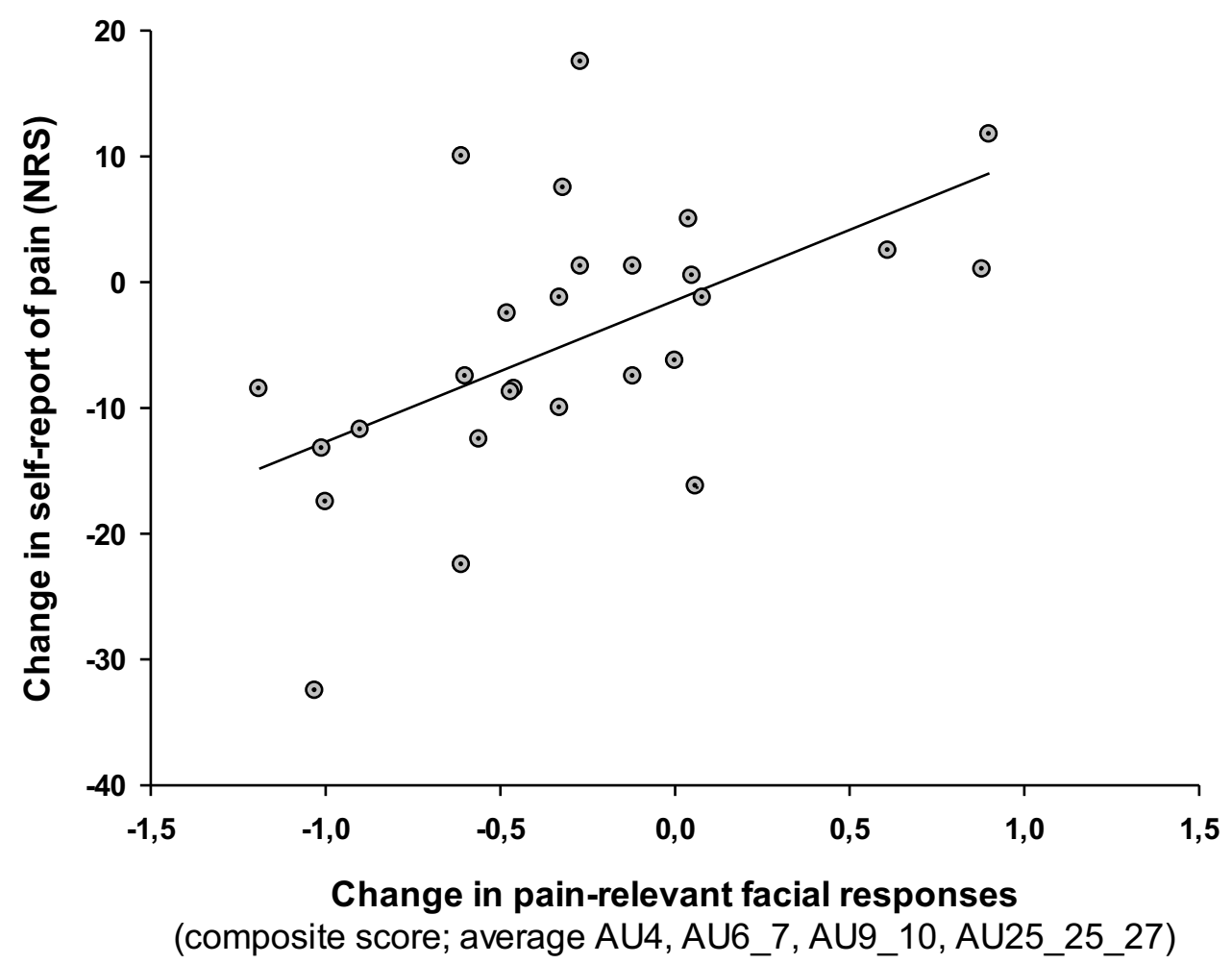

Figure 5 Correlation between CPM effects (change scores between baseline and CPM blocks) in the two pain response systems.

methodological modification allows for widening the scope of CPM applications to cases with altered or limited consciousness and vigilance, cognitive impairment, aphasic problems and dementia. The available electrophysiological alternatives quantifying the modulatory CPM effect on the test stimulus by assessing nociceptive flexion reflexes $^{23}$ or nociceptive brain potentials ${ }^{24-26}$ are limited to very brief stimuli with a very immediate onset that do not mirror most clinical pain states. The use of facial responses allows application of clinically more relevant stimuli and responses.

The inhibitory effects determined through recording the facial responses were statistically significant and were of comparable size as the inhibitory effects assessed by means of psychophysical parameters (ie NRS ratings) in the present study. The facial responses also clearly show the peculiarity of CPM inhibition, which leads - in most cases - to a reduction of pain indication although a second pain stimulus was added and the total noxious load is increased. Thus, our CPM probe using facial responses appeared to be as sensitive as the typical CPM paradigms (demonstrating the variability in the CPM effect, ranging from inhibition (in most cases) to facilitation), at least under the ideal conditions, which we created for this first time testing by studying only women (women are known to grade pain intensities by different facial responses very well $^{29}$ and were for that reason sampled in the present study).

Interestingly, the CPM effect shown for facial response was not redundant to the one shown for selfreport because the two modulatory effects were indeed significantly correlated but the correlation was only of moderate size allowing for the assumption of much unrelated variance. The relative independence of the "facial CPM" was also shown by the fact that statistically considering the variance of the "verbal CPM" effect did not abolish the large "facial CPM" effect. This finding relates well to the observation that although self-report ratings as well as facial responses to pain both reflect the intensity and the unpleasantness dimensions of pain, ${ }^{35,40}$ there are nevertheless some aspects of the multidimensional pain experiences that not are not fully captured by each type of response (e.g. ${ }^{28,35,41,42}$ ). Moreover, the self-report of pain is complex and is influenced as well as biased by higher mental processes. The facial response - in contrast - is a more automatic response that is less subject to voluntary control by higher mental processes. ${ }^{43}$ Given the more reflex-like nature of facial responses, facial CPM 
probes might even give more direct insights into the basics of CPM mechanisms.

It has been shown over the last two decades that there is sizeable inter-individual variance in the facial expression of pain, which is due to varying combinations of Action Units (AUs). ${ }^{27}$ The unifying backbone of these varying pain-relevant combinations seems to be AU 6_7 (contraction of the muscles surrounding the eyes; orbicularis oculi contraction). ${ }^{27,41}$ The orbicularis oculi contraction (AU 6_7) is the most frequent facial response to pain and may be combined with AU 4 (contraction of the eyebrows) or AU 9_10 (nose wrinkle/upper lip raise) or AU 25_26_27 (opened mouth) leading to the various pain faces known. ${ }^{44}$ Given that we found significant CPM inhibition in 3 out of the 4 pain-relevant AUs and given that AU 6_7 ("unifying backbone" of the pain faces) showed a moderate to large CPM effect suggests that despite the variability in facial responses to pain, CPM effects can be found regardless of the individual pain face. Our finding also suggests, that it might be sufficient to just focus on the contraction of the muscles surrounding the eyes (AU 6_7) in order to assess the endogenous inhibitory CPM effects in the face. Just focusing on one facial movement could make it easier to apply this method in research (eg assessment via EMG recordings or FACS analysis) and in clinical context.

If future studies can replicate our findings and show that the facial CPM effect is a reliable and valid alternative of assessing endogenous pain inhibition in humans, ${ }^{2,45}$ the scope of study can be widened to patients, who are compromised in verbal communication due to impairments of cognitive and linguistic skills or by restriction of vigilance and consciousness. ${ }^{46-48}$ However, verbal and non-verbal assessment of pain inhibition must not be seen as mutually exclusive alternatives. Instead, both can strengthen the sensitivity of a diagnostic probe for testing CPM effects also in vigilant and cognitively intact humans when assessed in parallel, given the unique information that each type contributes. ${ }^{43}$ Whereas the facial expression of pain can capture more bottom-up, automatic aspects of pain, the verbal report is easier to assess. Moreover, only relying on facial expression might hold the risk that mild pain is overlooked (given that the threshold of eliciting facial responses is often higher than the subjective pain threshold). The sample of the present study was deliberately biased to include only young women to create optimal conditions for this first time testing of CPM effects by recording the facial responses to the test stimulus. Since we were successful in verifying facial CPM effects in individuals, who are particularly apt in facially expressing pain in a graded fashion, ${ }^{29}$ replications in men and individuals with advanced ages ${ }^{49}$ are now the necessary next step. Since FACS coding is still a manual task that requires a lot of time for execution, we could not manage the study of both genders and further age groups at once. Moreover, in future studies, different CPM protocols could be tried out; ${ }^{50}$ eg applying the test stimuli after the end of the conditioning stimulus (sequential paradigm) to better separate the effect of distraction on facial and subjective pain responses from CPM effects.

\section{Conclusion}

We succeeded for the first to demonstrate modulatory CPM effects through recording of the facial response to the test stimuli instead of only using self-report ratings as a response measure. Given the clinical relevance of assessing CPM effects, the parallel assessment of verbal and facial CPM effects might be a promising approach with wider scope of applications and increased diagnostic robustness against biasing influences.

\section{Acknowledgment}

The study was supported by a research grant from the Deutsche Forschungsgemeinschaft (DFG, Ku2294/6).

\section{Disclosure}

The authors report no conflicts of interest for this work.

\section{References}

1. Yarnitsky D. Conditioned pain modulation (the diffuse noxious inhibitory control-like effect): its relevance for acute and chronic pain states. Curr Opinion Anesthesiology. 2010;23(5):611-615. doi:10.10 97/ACO.0b013e32833c348b

2. Lewis GN, Luke H, Rice DA, Rome K, McNair PJ. Reliability of the conditioned pain modulation paradigm to assess endogenous inhibitory pain pathways. Pain Res Manage. 2012;17(2):98-102. doi:10.1155/ 2012/610561

3. McPhee ME, Graven-Nielsen T. Recurrent low back pain patients demonstrate facilitated pronociceptive mechanisms when in pain, and impaired antinociceptive mechanisms with and without pain. Pain. 2019;160(12):2866-2876. doi:10.1097/j.pain.0000000000001679

4. Marcuzzi A, Chakiath RJ, Siddall PJ. Conditioned Pain Modulation (CPM) is Reduced in Irritable Bowel Syndrome. J Clin Gastroenterol. 2019;53(6):399-408. doi:10.1097/MCG.0000000000001181

5. Moana-Filho EJ, Babiloni AH, Theis-Mahon NR. Endogenous pain modulation in chronic orofacial pain: a systematic review and meta-analysis. Pain. 2018;159(8):1441-1455. doi:10.1097/j.pain.0000 000000001263

6. O'Brien AT, El-Hagrassy MM, Rafferty H, et al. Impact of therapeutic interventions on pain intensity and endogenous pain modulation in knee osteoarthritis: a systematic review and meta-analysis. Pain Med. 2019;20(5):1000-1011. doi:10.1093/pm/pny261 
7. Kisler LB, Granovsky Y, Coghill RC, et al. Do patients with interictal migraine modulate pain differently from healthy controls? A psychophysical and brain imaging study. Pain. 2018;159 (12):2667-2677. doi:10.1097/j.pain.0000000000001380

8. Stroemel-Scheder C, Karmann AJ, Ziegler E, et al. Sleep, experimental pain and clinical pain in patients with chronic musculoskeletal pain and healthy controls. J Pain Res. 2019;12:3381. doi:10.2147/ JPR.S211574

9. Pielsticker A, Haag G, Zaudig M, Lautenbacher S. Impairment of pain inhibition in chronic tension-type headache. Pain. 2005;118(1-2):215-223. doi:10.1016/j.pain.2005.08.019

10. Granovsky Y. Conditioned pain modulation: a predictor for development and treatment of neuropathic pain. Curr Pain Headache Rep. 2013;17(9):p.361. doi:10.1007/s11916-013-0361-8

11. Kong JT, Schnyer RN, Johnson KA, Mackey S. Understanding central mechanisms of acupuncture analgesia using dynamic quantitative sensory testing: a review. Evid Based Complement Altern Med. 2013;2013.

12. O’Brien AT, Deitos A, Pego YT, Fregni F, Carrillo-de-la-peña MT. Defective endogenous pain modulation in fibromyalgia: a meta-analysis of temporal summation and conditioned pain modulation paradigms. J Pain. 2018;19(8):819-836. doi:10.1016/j.jpain.20 18.01 .010

13. Edwards RR, Dolman AJ, Martel MO, et al. Variability in conditioned pain modulation predicts response to NSAID treatment in patients with knee osteoarthritis. BMC Musculoskelet Disord. 2016;17(1):1-9. doi:10.1186/s12891-016-1124-6

14. Horn-Hofmann C, Capito ES, Wolstein J, Lautenbacher S. Acute alcohol effects on conditioned pain modulation, but not temporal summation of pain. Pain. 2019;160(9):2063-2071. doi:10.1097/j. pain.0000000000001597

15. Kunz M, Scholl KE, Schu U, Lautenbacher S. GABAergic modulation of diffuse noxious inhibitory controls (DNIC): a test by use of lorazepam. Exp Brain Res. 2006;175(2):363-371. doi:10.1007/ s00221-006-0558-8

16. Yarnitsky D, Granot M, Nahman-Averbuch H, Khamaisi M, Granovsky Y. Conditioned pain modulation predicts duloxetine efficacy in painful diabetic neuropathy. Pain. 2012;153(6):1193-1198. doi:10.1016/j.pain.2012.02.021

17. Petersen KK, Vaegter HB, Stubhaug A, André W, Scammell BE. "The predictive value of quantitative sensory testing: a systematic review on chronic postoperative pain and the analgesic effect of pharmacological therapies in patients with chronic pain.". Pain. 2021;162(1):31-44. doi:10.1097/j.pain.0000000000002019

18. Ramaswamy S, Wodehouse T. Conditioned pain modulation-A comprehensive review. Neurophysiol Clin. 2020. doi:10.1016/j. neucli.2020.11.002

19. Hackett J, Naugle KE, Naugle KM. The Decline of Endogenous Pain Modulation With Aging: a Meta-Analysis of Temporal Summation and Conditioned Pain Modulation. J Pain. 2019;21(5-6):514-528. doi:10.1016/j.jpain.2019.09.005

20. Karmann AJ, Lautenbacher S, Kunz M. The role of inhibitory mechanisms in the regulation of facial expressiveness during pain. Biol Psychol. 2015;104:82-89. doi:10.1016/j.biopsycho.2014.11.016

21. Traxler J, Hanssen MM, Lautenbacher S, Ottawa F, Peters ML. General versus pain-specific cognitions: pain catastrophizing but not optimism influences conditioned pain modulation. Eur J Pain. 2019;23(1):150-159. doi:10.1002/ejp.1294

22. Martel MO, Wasan AD, Edwards RR. Sex differences in the stability of conditioned pain modulation (CPM) among patients with chronic pain. Pain Med. 2013;14(11):pp.1757-1768. doi:10.1111/pme.12220

23. Dhondt E, Van Oosterwijck S, Coppieters I, Danneels L, Van Oosterwijck J. Effects of Conditioned Pain Modulation on the Nociceptive Flexion Reflex in Healthy People. Clin J Pain. 2019;35 (9):794-807. doi:10.1097/AJP.0000000000000724
24. Albu S, Meagher MW. Divergent effects of conditioned pain modulation on subjective pain and nociceptive-related brain activity. Exp Brain Res. 2019;237(7):1735-1744. doi:10.1007/ s00221-019-05545-8

25. Ladouceur A, Rustamov N, Dubois JD, et al. Inhibition of pain and pain-related brain activity by heterotopic noxious counter-stimulation and selective attention in chronic non-specific low back pain. Neuroscience. 2018;387:201-213. doi:10.1016/j.neuroscience.2017. 09.054

26. Kunz M, Mohammadian P, Renner B, Roscher S, Kobal G, Lautenbacher S. Chemo-somatosensory evoked potentials: a sensitive tool to assess conditioned pain modulation? Somatosens Mot Res. 2014;31(2):100-110. doi:10.3109/08990220.2014.887562

27. Kunz M, Meixner D, Lautenbacher S. Facial muscle movements encoding pain —a systematic review. Pain. 2019;160(3):535-549. doi:10.1097/j.pain.0000000000001424

28. Kunz M, Mylius V, Schepelmann K, Lautenbacher S. On the relationship between self-report and facial expression of pain. J Pain. 2004;5 (7):368-376. doi:10.1016/j.jpain.2004.06.002

29. Kunz M, Gruber A, Lautenbacher S. Sex differences in facial encoding of pain. J Pain. 2006;7(12):915-928. doi:10.1016/j.jpain.2006. 04.012

30. Horn-Hofmann C, Kunz M, Madden M, Schnabel EL, Lautenbacher S. Interactive effects of conditioned pain modulation and temporal summation of pain - the role of stimulus modality. Pain. 2018;159(12):2641-2648. doi:10.1097/j.pain.0000000000001 376

31. Horn-Hofmann C, Priebe JA, Schaller J, Görlitz R, Lautenbacher S. Lack of predictive power of trait fear and anxiety for conditioned pain modulation (CPM). Exp Brain Res. 2016;234(12):3649-3658. doi:10.1007/s00221-016-4763-9

32. Sheehan DV, Lecrubier Y, Sheehan KH, et al. The Mini-International Neuropsychiatric Interview (MINI): the development and validation of a structured diagnostic psychiatric interview for DSM-IV and ICD-10. J Clin Psychiatry. 1998;59 Suppl 20:22-33; quiz 34-57.

33. Bär KJ, Köhler S, de la Cruz F, Schumann A, Zepf FD, Wagner G. Functional consequences of acute tryptophan depletion on raphe nuclei connectivity and network organization in healthy women. NeuroImage. 2020;207:116362. doi:10.1016/j.neuroimage.2019.116 362

34. Ekman P, Friesen WV. Facial Action Coding System: A Technique for the Technique for the Measurement of Facial Movement. Palo Alto, CA: Consulting Psychologists Press; 1978.

35. Kunz M, Chen JI, Rainville P. Keeping an eye on pain expression in primary somatosensory cortex. NeuroImage. 2020;217:116885. doi:10.1016/j.neuroimage.2020.116885

36. Karmann AJ, Lautenbacher S, Bauer F, Kunz M. The influence of communicative relations on facial responses to pain: does it matter who is watching? Pain Res Manage. 2014;19.

37. Karmann AJ, Lauer C, Ziegler E, Killian L, Horn-Hofmann C, Lautenbacher S. Associations of nocturnal sleep with experimental pain and pain catastrophizing in healthy volunteers. Biol Psychol. 2018;135:1-7. doi:10.1016/j.biopsycho.2018.02.015

38. Kunz M, Faltermeier N, Lautenbacher S. Impact of visual learning on facial expressions of physical distress: a study on voluntary and evoked expressions of pain in congenitally blind and sighted individuals. Biol Psychol. 2012;89(2):467-476. doi:10.1016/j. biopsycho.2011.12.016

39. Prkachin KM. The consistency of facial expressions of pain: a comparison across modalities. Pain. 1992;51(3):297-306. doi:10.10 16/0304-3959(92)90213-U

40. Kunz M, Lautenbacher S, LeBlanc N, Rainville P. Are both the sensory and the affective dimensions of pain encoded in the face? Pain. 2012;153(2):350-358. doi:10.1016/j.pain.2011.10.027 
41. Craig KD, Prkachin KM, Grunau RVE. The facial expression of pain. In: Turk DC, Melzack R, editors. Handbook of Pain Assessment. 2 ed. New York: Guilford; 2001:pp 153-169.

42. Kunz M, Chen JI, Lautenbacher S, Vachon-Presseau E, Rainville P. Cerebral regulation of facial expressions of pain. J Neurosci. 2011;31 (24):8730-8738. doi:10.1523/JNEUROSCI.0217-11.2011

43. Hadjistavropoulos T, Craig KD. A theoretical framework for understanding self-report and observational measures of pain: a communications model. Behav Res Ther. 2002;40(5):551-570. doi:10.1016/S0005-7967(01)00072-9

44. Kunz M, Lautenbacher S. The faces of pain: a cluster analysis of individual differences in facial activity patterns of pain. Eur $J$ Pain. 2014;18(6):pp.813-823. doi:10.1002/j.1532-2149.2013. 00421.x

45. Kennedy DL, Kemp HI, Ridout D, Yarnitsky D, Rice AS. Reliability of conditioned pain modulation: a systematic review. Pain. 2016;157 (11):p.2410. doi:10.1097/j.pain.0000000000000689

46. Defrin R, Amanzio M, De Tommaso M, et al. Experimental pain processing in individuals with cognitive impairment: current state of the science. Pain. 2015;156(8):1396-1408. doi:10.1097/j.pain.000 0000000000195
47. Hadjistavropoulos T, Browne ME, Prkachin KM, Taati B, Ashraf A Mihailidis A. Pain in severe dementia: a comparison of a fine-grained assessment approach to an observational checklist designed for clinical settings. Eur J Pain. 2018;22(5):915-925.

48. Lautenbacher S, Kunz M. Facial pain expression in dementia: a review of the experimental and clinical evidence. Curr Alzheimer Res. 2017;14 (5):501-505. doi:10.2174/1567205013666160603010455

49. Kunz M, Mylius V, Schepelmann K, Lautenbacher S. Impact of age on the facial expression of pain. J Psychosom Res. 2008;64 (3):311-318. doi:10.1016/j.jpsychores.2007.09.010

50. Yarnitsky D, Bouhassira D, Drewes AM. Recommendations on practice of conditioned pain modulation (CPM) testing. Eur $j$ Pain. 2015;19(6):805-806. doi:10.1002/ejp.605

\section{Publish your work in this journal}

The Journal of Pain Research is an international, peer reviewed, open access, online journal that welcomes laboratory and clinical findings in the fields of pain research and the prevention and management of pain. Original research, reviews, symposium reports, hypothesis formation and commentaries are all considered for publication. The manuscript management system is completely online and includes a very quick and fair peer-review system, which is all easy to use. Visit http:// www.dovepress.com/testimonials.php to read real quotes from published authors. 